\title{
Solutions of One-Dimensional Effective Mass Schrödinger Equation for PT-Symmetric Scarf Potential*
}

\author{
A.-P. ZHANG ${ }^{a \dagger}$ P. Shi, Y.-W. Ling AND Z.-W. HUA \\ Faculty of Science, Xi'an University of Architecture and Technology, Xi'an, 710055, People's Republic of China
}

(Received March 22, 2011; in final form August 3, 2011)

\begin{abstract}
The one-dimensional effective mass Schrödinger equation for $P T$-symmetric Scarf potential is investigated. The analytical expressions of energy eigenvalue and corresponding wave function are presented. They are accomplished by using an appropriate coordinate transformation to map the transformed exactly solvable one-dimensional Schrödinger equation with constant mass into the position-dependent mass equation. In the computation, three different forms of mass distributions are considered.
\end{abstract}

PACS: 03.65.Fd, 03.65.Ge, 03.65.-w

\section{Introduction}

In quantum mechanics, exact solutions of the wave equation are important to understand the concept of physics. Therefore, the exact solutions for the Schrödinger equation, the Klein-Gordon equation, the Dirac equation have been an important subject of theoretical research all along [1-5]. For many years people have developed many effective methods to solve the constant mass equation, such as supersymmetric (SUSYQM) [6], factor method [7], path integral representation [8], asymptotic iteration method [9], WKB [1], the Nikiforov-Uvarov (NU) method [10] etc.

Recently, there has been an increasing interest in the position-dependent-mass (or effective mass) Schrödinger equation for some physical potentials [11-15]. Its important applications are found in the fields of material science and condensed matter physics such as semiconductors [16], quantum dots [17], ${ }^{3} \mathrm{He}$ clusters [18], quantum liquids [19], semiconductor heterostructures [20], etc. Compared to the constant mass wave equation, the position-dependent-mass Schrödinger equation is more complex. It is difficult to obtain its analytical solution as usual. However, for some special potential, people have developed some available methods to obtain the analytical solutions for the effective mass

\footnotetext{
* Supported by Natural Science Basic Research Plan in Shaanxi Province of China (SJ08A13) and NSF of the Education Bureau of Shaanxi Province of China (O9jk534).

$\dagger$ corresponding author; e-mail: apzhang163@163.com
}

Schrödinger equation [11-14]. One of them is the coordinate transformation introduced by Gönül and Alhaidari [12-14]. The basic idea of the method [12-14] is that one can map the transformed exactly solvable one-dimensional Schrödinger equation with constant mass into the position-dependent mass equation by using an appropriate coordinate transformation. Therefore, the mapping result will give not only the energy spectra of the effective mass equation, but also the corresponding wave functions.

On the other hand, in Bender and Boettcher's work [21] it was shown that non-Hermitian Hamiltonian operator, such as $H=p^{2}+x^{2}(\mathrm{i} x)^{\varepsilon}(\varepsilon<0)$, possesses the real spectrum due to its parity-time symmetry $(P T$ symmetry), where $P$ and $T$ are the parity and time reversal operators, respectively. These operators are defined by acting on the position and momentum operators $x$ and $p[21]$ :

$$
\begin{aligned}
& P: x \rightarrow-x, \quad p \rightarrow-p, \\
& T: x \rightarrow x, \quad p \rightarrow-p, \quad \mathrm{i} \rightarrow-\mathrm{i} .
\end{aligned}
$$

The Hamiltonian will be $P T$-symmetric if

$$
[P T, H]=0 \text {. }
$$

Mostafazadeh has generalized PT symmetry to pseudo-Hermiticity. In fact, the Hamiltonian is considered to be $\eta$-pseudo Hermitian if $\mathrm{H}^{+}=\eta \mathrm{H}^{-1}[22]$. Non-Hermitian but $P T$-symmetry models have applications in different fields, such as optics [23], nuclear physics [24], condensed matter [25], the Berry phase [26] and population biology [27]. Recently, many researchers have studied the solutions of the relativistic and non-relativistic wave equation with some $P T$-symmetric potentials (for example: complexified Pöschl-Teller II potential model [28], 
real Rosen-Morse I potential model [29] and three-dimensional Eckart potential [30]). In Ref. [31], the author obtained a closed form expression of the $c$ operator for PT-symmetric Scarf I potential.

In this paper, we intend to study the bound state solutions of one-dimensional effective mass Schrödinger equation for $P T$-symmetry potential by using the coordinate transformation method. When the mass is dependent on position coordinate, PT-symmetric Hamiltonian meets the following conditions:

$$
m(x)=m(-x) \quad \text { and } \quad V^{*}(-x)=V(x) .
$$

Our aim, by considering three forms of the mass distributions, is to show the one-dimensional Schrödinger equation used in the position-dependent mass formalism. We obtained three new non-PT-symmetric and non-Hermitian potential functions by using the transformation. As a result, because of the exactly solvable property of the constant mass equation, three set of the energy eigenvalues of the bound states and corresponding wave functions for three different effective mass distributions are obtained.

The paper is organized as follows. In Sect. 2, we briefly introduce the basic concept of the coordinate transformation method in Ref. [14]. In Sect. 3, as an example of $P T$-symmetric Scarf potential, we consider to apply the coordinate transformation method to obtain the energy eigenvalues of the bound states and corresponding wave functions for three different effective mass distributions. Results are discussed in Sect. 4.

\section{Coordinate transformation method}

When the effective mass depends on the spatial position, because of the noncommutation between mass operator and momentum operator, the kinetic energy operator of the system has many definitions. In this paper, the position-dependent-mass Hamiltonian is defined [14] as

$$
\begin{aligned}
H & =P \frac{1}{2 M(x)} P+V(x) \\
& =-\frac{\hbar^{2}}{2 m_{0}}\left(\nabla \frac{1}{m(x)} \nabla\right)+V(x),
\end{aligned}
$$

where $m(x)$ and $V(x)$ are real functions of the configuration space coordinates. Using atomic units $\hbar=$ $2 m_{0}=1$, the Hamiltonian results in the following time-independent wave equation in one dimension:

$$
\left(\partial_{x} \frac{1}{m(x)} \partial_{x}-V(x)\right) \psi(x)=-E \psi(x),
$$

where $E$ and $\psi(x)$ are the energy spectrum and wave function with the position-dependent mass system, respectively.

On the other hand, the one-dimensional constant mass wave equation is

$$
\left(\frac{\mathrm{d}^{2}}{\mathrm{~d} y^{2}}-V(y)\right) \phi(y)=-\varepsilon \phi(y),
$$

where $V(y)$ is the potential function, and $\varepsilon$ is the energy spectrum. Defining a transformation $y \rightarrow x$ for a mapping $y=f(x)$, we rewrite the wave functions in the form of

$$
\phi(y)=g(x) \psi(x) .
$$

Thus, the transformed Schrödinger equation takes

$$
\begin{gathered}
\psi^{\prime \prime}(x)+\left(2 \frac{g^{\prime}(x)}{g(x)}-\frac{f^{\prime \prime}(x)}{f^{\prime}(x)}\right) \psi^{\prime}(x) \\
+\left(\frac{g^{\prime \prime}(x)}{g(x)}-\frac{g^{\prime}(x)}{g(x)} \frac{f^{\prime \prime}(x)}{f^{\prime}(x)}\right) \psi(x) \\
-f^{\prime}(x)^{2}[V(y)-\varepsilon] \psi(x)=0 .
\end{gathered}
$$

Comparing with Eq. (5) we obtain the following conditions on the transformation:

$$
\begin{aligned}
& g(x)=\left(\frac{f^{\prime}(x)}{m(x)}\right)^{1 / 2}, \\
& V(x)-E=\frac{f^{\prime}(x)^{2}}{m(x)}[V(y)-\varepsilon] \\
& \quad+\frac{1}{2 m(x)}\left[F(m(x))-F\left(f^{\prime}(x)\right)\right],
\end{aligned}
$$

where $F(z)=\frac{z^{\prime \prime}}{z}-\frac{3}{2}\left(\frac{z^{\prime}}{z}\right)^{2}$. For a constant mass problem, the transformation is $y=x$. In this case, $g(x)=1$. Thus Eq. (8) will be recovered to Eq. (6).

It is straightforward to find that the coordinate transformation can construct the relationship between the constant mass and position-dependent mass equation. Given a spatially dependent mass $m(x)$, if we can choose the appropriate transformation function $y=f(x)$ to determine $g(x)$, as given by Eq. (9), and we will use Eq. (10) to deduce the energy spectra $E_{n}$, and the potential function $V(x)$ for the position-dependent-mass wave equation. This is because the potential should be independent of the index $n$. As a result, according to Eq. (7), we will obtain wave functions for the position-dependent-mass Schrödinger equation.

\section{PT-symmetric Scarf potential}

The Scarf potential in one dimension is

$$
\begin{aligned}
V(y) & =-U_{0} \operatorname{sech}^{2} \frac{y}{a}+U_{1} \operatorname{sech} \frac{y}{a} \tanh \frac{y}{a}, \\
U_{0} & >0, \quad U_{1} \neq 0,
\end{aligned}
$$

which is a hyperbolic potential. Here, the parameters $U_{0}$ and $U_{1}$ describe the depth of the potential well; $a$ is a parameter to control the width of the potential well. For $U_{0}=85, U_{1}=100$ and $a=1$, the potential is shown in Fig. 1.

Here our main goal is to study the solutions of one-dimensional Schrödinger equation for $P T$-symmetric 


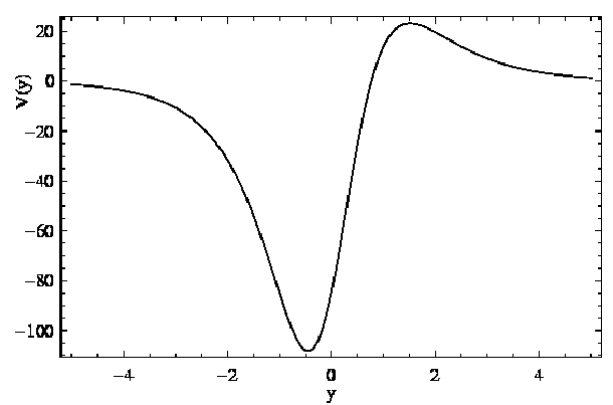

Fig. 1. Scarf potential for $U_{0}=85, U_{1}=100$ and $a=1$.

Scarf potential. For PT-symmetric and non-Hermitian potential case, we choose the parameters $U_{0}$, and $U_{1}$ are arbitrarily real, and $a$ is taken to be a complex parameter (i.e., $1 / a \rightarrow \mathrm{i} / a$ ). Consequently, the potential is transformed into the form

$$
\begin{aligned}
V(y) & =-U_{0} \sec ^{2} \frac{y}{a}+\mathrm{i} U_{1} \sec \frac{y}{a} \tan \frac{y}{a}=V^{*}(y), \\
U_{0} & >0, \quad U_{1} \neq 0 .
\end{aligned}
$$

Equations (11) and (12) are dramatically different. We note that the transformed potential in Eq. (12) has a trigonometric and periodic form. In Fig. 2 we plot real and imaginary part PT-symmetric Scarf potential for $U_{0}=85, U_{1}=100$ and $a=1$, where $\operatorname{Re}(V(y))=$ $-U_{0} \sec ^{2} \frac{y}{a}, \operatorname{Im}(V(y))=U_{1} \sec \frac{y}{a} \tan \frac{y}{a}$. Figure 2 shows that the $P T$-symmetric Scarf potential is periodic, and there exists real energy spectrum due to unbroken $P T$ -symmetry [21].

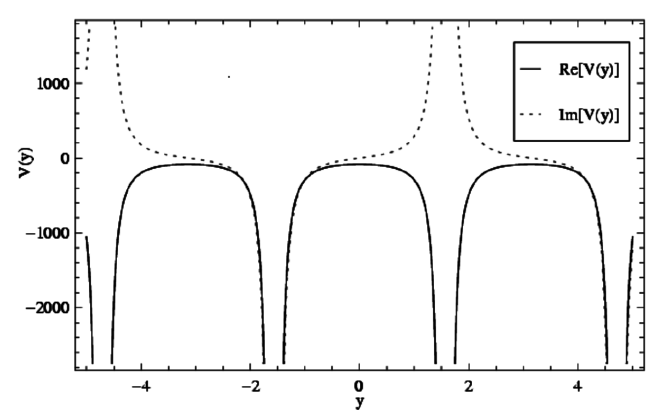

Fig. 2. Real and imaginary part $P T$-symmetric Scarf potential for $U_{0}=85, U_{1}=100$ and $a=1$, where $\operatorname{Re}(V(y))=-U_{0} \sec ^{2} \frac{y}{a}, \operatorname{Im}(V(y))=U_{1} \sec \frac{y}{a} \tan \frac{y}{a}$.

The constant-mass Schrödinger equation is

$$
\left(-\frac{\mathrm{d}^{2}}{\mathrm{~d} y^{2}}+V(y)\right) \phi(y)=\varepsilon \phi(y)
$$

where the natural atomic units $\hbar=2 m_{0}=1$ are chosen. Setting the following notation:

$$
\eta=\frac{\sqrt{\left(1-4 U_{0} a^{2}\right)^{2}+16 U_{1}^{2} a^{4}}}{8},
$$

we have solved the Schrödinger equation for the constant-mass potential $V(y)$ (i.e. Eq. (12)) via the NikiforovUvarov method [10]. The energy eigenvalues are obtained as follows:

$$
\begin{aligned}
\varepsilon_{n} & =\frac{1}{a^{2}}\left(\frac{1}{2}+n^{2}+n-(2 n+1) \sqrt{\eta-\frac{1-4 U_{0} a^{2}}{8}}\right. \\
& \left.-\eta-\frac{1+4 U_{0} a^{2}}{8}\right)
\end{aligned}
$$

where

$$
n=0,1,2 \ldots \leq(1+\sqrt{2}) \sqrt{\eta-\frac{1-4 U_{0} a^{2}}{8}}-\frac{1}{2},
$$

which means that the number of real eigenvalues is finite. When

$$
n=\left(2 \sqrt{\eta-\frac{1-4 U_{0} a^{2}}{8}}-1\right) / 2,
$$

there exists the lowest eigenvalue. The $P T$-symmetric Hamiltonian has real spectra, which means that $P T$ -symmetric spontaneous breakdown cannot be implemented.

The corresponding unnormalized wave functions are obtained

$$
\begin{aligned}
& \phi_{n}(y)=\left(1-\sin \frac{y}{a}\right)^{\frac{2 \alpha+1}{4}}\left(1+\sin \frac{y}{a}\right)^{\frac{2 \beta+1}{4}} \\
& \times P_{n}^{(\alpha, \beta)}\left(\sin \frac{y}{a}\right)
\end{aligned}
$$

where $P_{n}^{(\alpha, \beta)}(x)$ is the Jacobi polynomials [32], and

$$
\begin{aligned}
& \alpha=\sqrt{\eta+\frac{1-4 U_{0} a^{2}}{8}}+\mathrm{i} \sqrt{\eta-\frac{1-4 U_{0} a^{2}}{8}}, \\
& \beta=\sqrt{\eta+\frac{1-4 U_{0} a^{2}}{8}}-\mathrm{i} \sqrt{\eta-\frac{1-4 U_{0} a^{2}}{8}} .
\end{aligned}
$$

Now, we study exact analytical solutions of the Schrödinger equation for $P T$-symmetric Scarf potential when the effective mass varies with the spatial position. We have chosen three different position-dependent-mass distributions in order to meet PT symmetry (i.e. Eq. (3)).

$$
\text { 3.1. Mass distribution } m(x)=\delta x^{-2}
$$

Firstly, we consider the mass distribution

$$
m(x)=\delta x^{-2},
$$

where $\delta$ is an arbitrary non-zero real constant coefficient. After analysis, we choose the following transformation function, which can divide the right-hand side of Eq. (10) into two parts

$$
f(x)=a \ln x .
$$

Substituting it into Eq. (10), we obtain the following effective potential:

$$
V(x)=-\frac{a^{2}}{\delta} U_{0} \sec ^{2}(\ln x)
$$




$$
+\mathrm{i} \frac{a^{2}}{\delta} U_{1} \sec (\ln x) \tan (\ln x) .
$$

which is a non- $P T$-symmetric, non-Hermitian potential. Thus, the energy eigenvalue equation is obtained from Eqs. (10) and (21) as

$$
E_{n}=\frac{a^{2}}{\delta} \varepsilon_{n}+\frac{1}{4 \delta},
$$

which means that the energy levels are always real. Finally, we obtained the unnormalized wave functions as

$$
\begin{aligned}
& \psi_{n}(x)=\left(\frac{a x}{\delta}\right)^{-\frac{1}{2}}[1-\sin (\ln x)]^{\frac{2 \alpha+1}{4}}[1+\sin (\ln x)]^{\frac{2 \beta+1}{4}} \\
& \quad \times P_{n}^{(\alpha, \beta)}[\sin (\ln x)] .
\end{aligned}
$$

It can be seen that not only the energy spectra are always real when

$$
n=0,1,2 \ldots \leq(1+\sqrt{2}) \sqrt{\eta-\frac{1-4 U_{0} a^{2}}{8}}-\frac{1}{2},
$$

but also the lowest energy level varies with the mass distribution in the formula (19). Equation (23) shows that the mass distribution of the particle contributes to the eigenfunctions.

\subsection{Mass distribution $m(x)=\frac{\delta}{1+x^{2}}$}

We choose the second mass distribution

$$
m(x)=\frac{\delta}{1+x^{2}} .
$$

When

$$
f(x)=a \operatorname{arcsinh} x,
$$

substituting it into above equation, we obtain the following form:

$$
\left\{\begin{array}{l}
V(x)=-\frac{a^{2}}{\delta}\left[U_{0} \sec ^{2}(\operatorname{arcsinh} x)-\mathrm{i} U_{1} \sec (\operatorname{arcsinh} x)\right. \\
\quad \times \tan (\operatorname{arcsinh} x)]-\frac{1}{4 \delta\left(1+x^{2}\right)}, \\
E_{n}=\frac{a^{2}}{\delta} \varepsilon_{n}+\frac{1}{4 \delta} \\
\psi_{n}(x)=\left[\frac{a\left(1+x^{2}\right)^{\frac{1}{2}}}{\delta}\right]^{-\frac{1}{2}}[1-\sin (\operatorname{arcsinh} x)]^{\frac{2 \alpha+1}{4}} \\
\quad \times[1+\sin (\operatorname{arcsinh} x)]^{\frac{2 \beta+1}{4}} P_{n}^{(\alpha, \beta)}[\sin (\operatorname{arcsinh} x)] .
\end{array}\right.
$$

It can be seen that the effective potential is non- $P T$ -symmetric, non-Hermitian, but the eigenvalues are always real, and the eigenfunctions are dependent on the mass distribution of the particle.

$$
\text { 3.3. Mass distribution } m(x)=\frac{\delta}{\left(1+x^{2}\right)^{2}}
$$

When

$$
f(x)=a \arctan x,
$$

then the solutions of the position-dependent-mass equation are described by the following:

$$
\begin{aligned}
& V(x)=-\frac{a^{2}}{\delta} U_{0}\left(1+x^{2}\right)-\frac{2 x^{2}}{\delta}+\mathrm{i} \frac{a^{2}}{\delta} U_{1} x \sqrt{1+x^{2}}, \\
& E_{n}=\frac{a^{2}}{\delta} \varepsilon_{n}+\frac{1}{\delta},
\end{aligned}
$$

$$
\begin{gathered}
\psi_{n}(x)=\left[\frac{a}{\delta}\left(1+x^{2}\right)\right]^{-\frac{1}{2}}\left(1-\frac{x}{\sqrt{1+x^{2}}}\right)^{\frac{\alpha}{2}} \\
\times\left(1+\frac{x}{\sqrt{1+x^{2}}}\right)^{\frac{\beta}{2}} P_{n}^{(\alpha, \beta)}\left(\frac{x}{\sqrt{1+x^{2}}}\right) .
\end{gathered}
$$

From Eq. (28), we also find that the eigenvalues are always real, and the eigenfunctions are dependent on the mass distribution of the particle.

\section{Conclusion}

In this paper, we have investigated the one-dimensional Schrödinger equation for $P T$-symmetry Scarf potential with the effective mass distribution dependent on spatial position. The exactly solvable one-dimensional constant mass Schrödinger equation has been transformed into the form similar to the effective mass by using the appropriate coordinate transformation. In the computations, three different effective mass distributions are used. Thus three new non- $P T$-symmetric and non-Hermitian complex potentials are produced. The results of the transformation provide us the required energy spectra and wave functions for $P T$-symmetry Scarf potential. We find that for each mass distribution, not only the energy spectra are always real when

$$
n=0,1,2 \ldots \leq(1+\sqrt{2}) \sqrt{\eta-\frac{1-4 U_{0} a^{2}}{8}}-\frac{1}{2},
$$

but also the lowest energy level varies with the mass distribution of the particle. We also find that the eigenfunctions are dependent on the mass distribution of the particle. This work contributes to understand further the principles of quantum mechanics.

\section{Acknowledgments}

The authors wish to thank the anonymous referees for their valuable comments and suggestions.

\section{References}

[1] L.D. Landau, E.M. Lifshitz, Quantum Mechanics, 3rd ed., Pergamon Press, London 1977.

[2] S. Flügge, Practical Quantum Mechanics, Springer-Verlag, Berlin 1974.

[3] B.G. Bagrov, D.M. Gitman, Exact Solutions of Relativistic Wave Equations, Kluwer Academic Publisher, London 1990.

[4] W.C. Qiang, J.Y. Wu, S.H. Dong, Phys. Scr. 79, 065011 (2009).

[5] A.P. Zhang, W.C. Qiang, High Energy Nucl. Phys. 31, 1027 (2007).

[6] R. Dutt, A. Gangopadhyaya, U.P. Sukhatme, Am. J. Phys. 65, 400 (1997).

[7] L. Infeld, T.E. Hull, Rev. Mod. Phys. 23, 21 (1951).

[8] I. Sökmen, Phys. Lett. A 115, 249 (1986). 
[9] H. Ciftci, R.L. Hall, N. Saad, J. Phys. A, Math. Gen. 36, 11807 (2003).

[10] A.F. Nikiforov, V.B. Uvarov, Special Function of Mathematical Physics, Birkhauser, Basel 1988.

[11] J.M. Levy-Leblond, Phys. Rev. A 52, 1845 (1995).

[12] B. Gönül, D. Tutcu, O. Ozer, Mod. Phys. Lett. A 17, 2057 (2002)

[13] B. Gönül, O. Ozer, F. Üzgün, Mod. Phys. Lett. A 17, 2453 (2002).

[14] A.D. Alhaidari, Phys. Rev. A 66, 042116 (2002).

[15] C.Y. Cai, Z.Z. Ren, G.X. Ju, Acta Phys. Sin. 54, 2528 (2005).

[16] G. Bastard, Wave Mechanics Applied to Semiconductor Heterostructure, Les Edition de Physique, Les Ulis, France 1988.

[17] L. Serra, E. Lipparini, Europhys. Lett. 40, 667 (1997).

[18] M. Barranco, M. Pi, S.M. Gatica, E.S. Hernandez, J. Navarro, Phys. Rev. B 56, 8997 (1997).

[19] F. Arias de Savedra, J. Boronat, A. Polls, A. Fabrocini, Phys. Rev. B 50, 4248 (1997).

[20] C. Weisbuch, B. Vinter, Quantum Semiconductor Heterostructure, Academic Press, New York 1993.

[21] C.M. Bender, S. Boettcher, Phys. Rev. Lett. 80, 5243 (1998).
[22] Z. Ahmed, Phys. Lett. A 290, 19 (2001).

[23] R.N. Deb, A. Khare, B.D. Roy, Phys. Lett. A 307 215 (2003).

[24] N. Hatano, D.R. Nelson, Phys. Rev. B 56, 8651 (1997).

[25] D.R. Nelson, N.M. Shnerb, Phys. Rev. E 58, 1383 (1998).

[26] J.B. Gong, Qing-hai Wang, Phys. Rev. A 82, 012103 (2010).

[27] N.N. Bogolubov, A.A. Logunov, I.I. Todorov, Introduction to Axiomatic Field Theory, Benjamin, New York 1975.

[28] C.S. Jia, Y. Sun, Y. Li, Phys. Lett. A 305, 231 (2002).

[29] G. Lévai, Phys. Lett. A 372, 6484 (2008).

[30] A.P. Zhang, W.C. Qiang, Y.W. Ling, Chin. Phys. Lett. 26, 100302-1 (2009).

[31] R. Roychoudhury, P. Roy, J. Phys. A, Math. Theor. 40, F617 (2007).

[32] N.N. Lebedev, Special Function and Their Application, Prentice-Hall, Englewood Cliffs, NJ 1965. 